\title{
Coherent pore-scale imaging: phase contrast and ptychography
}

Igor Artyukov, Nikolai Popov, Alexander Vinogradov

Igor A. Artyukov, Nikolai L. Popov, Alexander V. Vinogradov, "Coherent porescale imaging: phase contrast and ptychography," Proc. SPIE 11886, International Conference on X-Ray Lasers 2020, 118860 (8 July 2021); doi: $10.1117 / 12.2593242$

SPIE. Event: XVII International Conference on X-Ray Lasers, 2020, Online Only 


\title{
Coherent pore-scale imaging: phase contrast and ptychography
}

\author{
Igor A. Artyukov", Nikolai L. Popov, Alexander V. Vinogradov \\ P.N. Lebedev Physical Institute, 53 Leninsky Prospekt, Moscow, Russia 119991
}

\begin{abstract}
The paper deals with an analytical study of the problem of pore detection and certification in bulk materials by means of $\mathrm{X}$-ray radiography. The optimum thickness of a sample under X-ray absorption investigation of the pores is found, that can be used for an improvement of the signal-to-noise ratio by the proper X-ray photon energy. In the case of low absorption an X-ray coherent beam can be used for production of phase contrast in the radiographic experiments. We present a simple model to calculate the complex value of the wave field formed by the sample. The model includes two dimensionless parameters: the Fresnel number $\mathrm{F}=a^{2} /(\lambda z)$, where $a$ is the pore radius, $\lambda$ is the wavelength, $\mathrm{z}$ is the sampleto-detector distance and the phase number $\Phi=a k \delta$, where $k=2 \pi \lambda$ and $\delta$ is the decrement of the real part of material's relative permittivity. The detailed analysis of the field structure is given with an estimation of the optimal position of the detector. The numerical simulation results are presented as well, which were obtained for the Gaussian type of the pore shape function. The stationary phase method of higher orders has been proven to simplify the Fresnel integral. The developed qualitative visualization of the pores with the help of phase contrast X-ray imaging complements other modern methods of monitoring porous-sensitive materials.
\end{abstract}

Keywords: X-ray imaging, pores, laser ceramics, phase contrast imaging, ptychography

\section{INTRODUCTION}

A presence of pores can change properties of the materials. The porosity effects can be natural or artificial, useful or harmful. Some studies and applications require an intensive saturation of the materials with the pores ${ }^{1}$. On the contrary, a production of laser ceramics needs a careful removal of the micropores ${ }^{2}$. A study of the pores is known to be both fundamental and practical .X-ray porosimetry as a part of general porosimetry $\mathrm{y}^{3-5}$ is a fast developing technique based on $\mathrm{X}$-ray small angle scattering ${ }^{6}$ and X-ray imaging. At present it profits from current advances in X-ray source development (new generation of synchrotron radiation and laboratory high brightness X-ray sources) as well as of the state-of-the-art X-ray imaging methods, such as phase contrast tomography ${ }^{8}$ and ptychography ${ }^{9}{ }^{10}$. At the same time, the image formation of the pores needs a more accurate and effective analytical description of the related phase contrast effects.

The goal of this paper is to present two analytical approaches to X-ray imaging of the pores developed for the cases of absorption and phase contrast experiments. A simplified model of porous objects makes it possible to formulate and optimize more general conditions of the experimental layout including the X-ray source parameters, sample dimensions and measurement geometry.

\section{THE ABSORPTION CONTRAST}

The main parameters and typical experimental layout are shown in Fig. 1. As an X-ray parallel beam running through the object under investigation, the measurement compares the number of photons going through the pore with then number of photons propagating through the bulk material without the pores. Considering the number of incident photons as a discrete random process one can write the signal-to-noise ratio $S N R$ as:

$$
S N R=\frac{\left|N_{a}-N_{b}\right|}{\sqrt{D\left\{N_{a}-N_{b}\right\}}}
$$

where $N_{b}$ is the number of output photons passed through the bulk material with no pores, $N_{a}$ is the number of output photons that met a pore of the radius $a$ and $D$ is the variance. For Poisson photon statistics the variance of two quantities is known to equal the sum of the variances, with each of them being equal to its average. Then Eq. (1) takes the form (see Fig.1):

*iart@lebedev.ru; phone 7499 132-6522; fax 7499 135- 7880; www.lebedev.ru 


$$
S N R=\frac{\left|N_{a}-N_{b}\right|}{\sqrt{D\left\{N_{a}-N_{b}\right\}}}=\frac{\left|N_{a}-N_{b}\right|}{\sqrt{N_{a}+N_{b}}}=\sqrt{N e^{-\mu l}} \frac{e^{\mu a}-1}{\sqrt{e^{\mu a}+1}}
$$

where $N$ is the total number of photons incident on the pore cross section and $\mu$ is the linear attenuation coefficient of the material. Eq. (2) yields the following formula for the number of incident photons $N$ :

$$
N=(S N R)^{2} e^{\mu l} \frac{e^{\mu a}+1}{\left(e^{\mu a}-1\right)^{2}}
$$

This expression can be used for choosing optimum values of the photon energy and the sample thickness. By introducing $x=e^{\mu a}$ and $y=l / a$ we have:

$$
\frac{N}{(S N R)^{2}}=x^{y} \frac{x+1}{(x-1)^{2}}
$$

The minimum of this value is reached at

$$
x=\frac{\sqrt{4 y^{2}-4 y+9}+3}{2(y-1)}
$$

For sample thickness much larger than the pore size $l>>a$ :

$$
x=1+\frac{2}{y}, y \gg 1 \text { and, hence, } \mu l=y \cdot \ln x \approx 2
$$

i.e. for the pore imaging the optimum optical thickness of a sample is $\mu l \approx 2$. Substituting this value into (4) one can obtain a simple formula for the total number of incident photons $N$ required for the pore image production with given SNR value:

$$
N=(S N R)^{2} \frac{e^{2}}{2} \frac{l^{2}}{a^{2}} \approx 100 \frac{l^{2}}{a^{2}}
$$

Here we use the practical value $(S N R)^{2} \approx 30$ (see Ref. ${ }^{7}$ ).

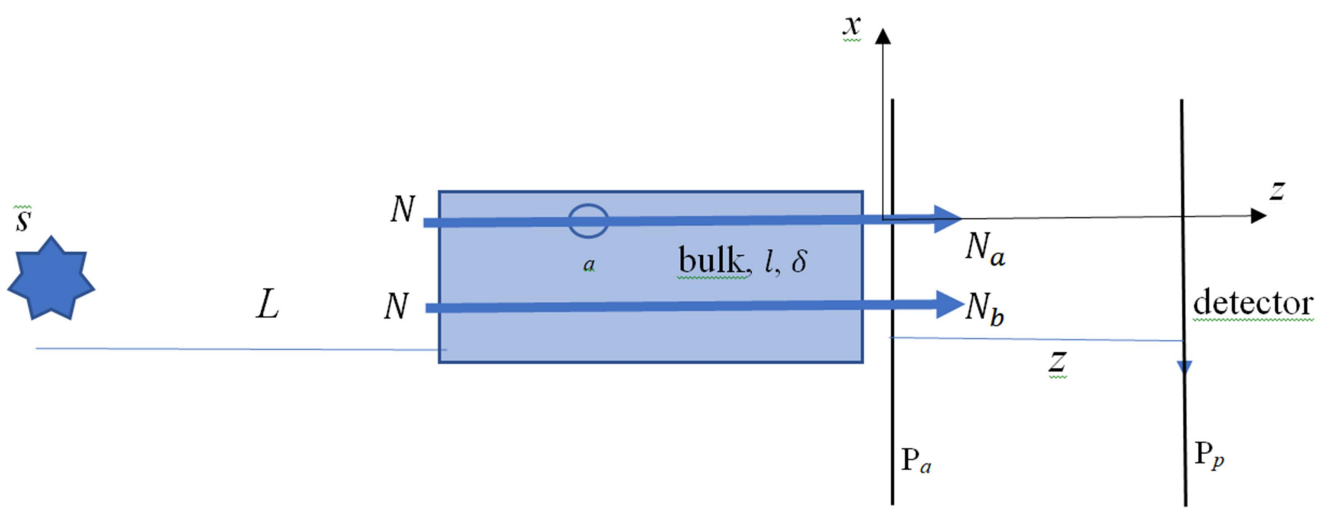

Figure 1. Geometry and main parameters of X-ray pore imaging: $l$-sample thickness, $a$ - pore radius, $\delta$ - decrement of the real part of material relative permittivity, $s$ - source size, $L$ - source to sample distance, $z$ - sample to detector distance.

As expected, the source flux needed for a clear pore imaging is proportional to the inverse square of the pore size. The flux can be decreased by an appropriate choice of the probing photon energy to reduce the sample thickness down to $l \approx$ $2 / \mu$ (see (6)). On the other hand, the smallest value of the thickness $l$ in (7) is limited by the mentioned condition $l>>a$ and restrictions of a sample manufacturing. In fact, formula (7) is an estimate of the number of photons per pixel in the assumption of pixel size being close to the pore radius $a$. The expression (7) multiplied by the total number of pixels gives us the total number of photons on the detector. 


\section{THE PORE X-RAY IMAGING AS A PHASE CONTRAST PROBLEM}

For the sake of completeness and brevity we present only the main formulas of phase contrast imaging in application to a detection and measurement of the pores in almost transparent materials. The general theory and other applications of phase contrast X-ray imaging can be found in many papers and textbooks ${ }^{7,11,12}$.

Let us consider the phase contrast imaging while neglecting the absorption. Then a ray passing through a pore in comparison with a neighboring ray outside the pore acquires the following phase difference (see Fig.1):

$$
S(x)=\begin{gathered}
0, \quad|x| \geq a, \\
-\delta k \sqrt{a^{2}-x^{2}},|x| \leq a,
\end{gathered}
$$

where $k$ is the wave vector, $x$ is the impact parameter of the ray relative to the pore center, $\delta$ is the decrement of the real part of material relative permittivity:

$$
\varepsilon=1-\delta+i \beta, \text { where } \delta, \beta \ll 1 ; \quad \mu=k \beta .
$$

With using the phase difference $S(x)$ one can write the wave field $u_{0}(x)$ right after the sample as:

$$
u_{0}(x)=e^{i S(x)} .
$$

In general, we will consider more common expression for the phase difference $S(x)$ instead of (8):

$$
S(x)=-\delta k a \varphi(\xi),-\infty<\xi<\infty, \xi=\frac{x}{a}
$$

where $\varphi(\xi)$ is called the pore shape function and it is a smooth function of $\xi=x / a$ at the interval $-\infty<\xi<\infty$ with the maximum value $\varphi_{\max }(\xi)=1$ at $\xi=0$.

Then, according to well-known Fresnel propagation theory the field $u(x, z)$ in the half space $z>0$ can be written as:

$$
u(x, z)=\sqrt{\frac{k}{2 \pi i z}} \int_{-\infty}^{\infty} u_{0}(s) e^{i \frac{k(s-x)^{2}}{2 z}} \mathrm{~d} s=\sqrt{\frac{k}{2 \pi i z}} \int_{-\infty}^{\infty} \exp \left\{i \frac{k(s-x)^{2}}{2 z}-i \delta k a \varphi\left(\frac{s}{a}\right)\right\} \mathrm{d} s
$$

or:

$$
u(x, z)=\sqrt{\frac{F}{i}} J(F, \Phi, \xi), J(F, \Phi, \xi)=\int_{-\infty}^{\infty} \exp \left\{i F \cdot \pi(t-\xi)^{2}-i \Phi \cdot \varphi(t)\right\} \mathrm{d} t, \xi=\frac{x}{a}
$$

where $F$ is the Fresnel number and $\Phi$ is the phase number, which takes into account the pore radius $a$ and the decrement of the real part of relative permittivity $\delta$ :

$$
F=\frac{k a^{2}}{2 \pi z}=\frac{a^{2}}{\lambda z}, \Phi=\delta k a .
$$

We suggest to express the space field coordinates $x$ and $z$ in (13) in the terms of dimensionless variables $\xi=x / a$ and $F=$ $a^{2} /(\lambda z)$, where $a$ is the pore radius and $a^{2} / \lambda$ is the distance that is of the order of the Rayleigh length, and the phase number $\Phi$ which represents the parameters of the object.

The question is: how the measurement of the field distribution (13) can be used for a detection of pore spaces inside nearly transparent samples? To address this question we study the properties of the Fresnel integral $J(F, \Phi, \xi)$ and functions $F$ and $\Phi$ in Appendix II. The corresponding asymptotic expansion of related fast oscillating integrals is considered in Appendix I.

The Fresnel integral $J(F, \Phi, \xi)$ carries the information about the pore spaces. It has two critical points: $t_{1}=\xi$ and $t_{2}=0$. This fact allows us to use the stationary phase method in the case of large values of $F$ and $\Phi$ (see Appendix II for our analysis in the case of small values of Fresnel and phase numbers). It is based on application of the perturbation theory at large distance $z$ and/or small values of $\delta$.

Usually microscopy and similar imaging techniques deal with $2 \mathrm{D}$ or $3 \mathrm{D}$ intensity distribution that is proportional to the square of field module $|u(x, z)|^{2}$. In this paper we expand the field amplitude $u(x, z)$ into the Taylor series or asymptotic series: $(x, z)=u^{(0)}(x, z)+u^{(1)}(x, z)+\cdots$. Obviously, in the case of phase contrast imaging of the pores the module of the first term in the series is nearly equal to 1 , that corresponds to the initial and/or non-perturbed field, 
$\left|u^{(0)}(x, z)\right|^{2}=$ and presents no useful information about the object strcuture. Therefore, to study of the pore space images or diffraction patterns one should take into account next two or more terms of the expansion series, with the third and fourth derivatives of the phase $S(x)$ being used (see Appendixes I and II). The mentioned mathematical expressions are utilized in Section 4 for analysis of the output field $u(x, z)$ formed by the sample.

\section{DEPENDENCE OF SCATTERED FIELD STRUCTURE $u(x, z)$ ON THE FRESNEL AND PHASE NUMBERS $F$ AND $\Phi$}

For a transparent material our concept of pore study can be summarized as follows (see also (8) - (13) and Section 3). The primary source of the information is the intensity measurement of the field in a plane after the sample (see Fig 1). So, the plane $P_{a}$ can be located right after the sample, where the field is not affected strongly by further diffraction in free space. In this case the object structure visualization can be performed only by the absorption contrast, since a change of the diffraction pattern inside the boundaries of the sample volume may be neglected. It should be mentioned that the field intensity distribution on the $P_{a}$ plane can be magnified using an optical system.

As the detector moves away from $P_{a}$ plane to far $P_{p}$ plane, the inline phase contrast enhances and forms a clear diffraction pattern. In this case a processing of such experimental can be carried out with the help of either analytical or numerical simulation of the wave propagation from the source through the sample and then in free space from the plane $P_{a}$ to the detector plane $P p^{13,14}$.

In this paper we show that it is possible to calculate the amplitude and phase distributions of the field in both planes $P_{a}$ and $P_{p}$ from the intensity measured on the plane $P_{p}$ with using iterative computer procedures. Obviously, our method of coherent imaging is based on the phase retrieval algorithms and principles of in-line holography, coherent diffraction imaging and ptychography ${ }^{7,9}, 15$. Our previous publication ${ }^{16}$ presents general discussion on the choice of practical geometry and computer domains. In this paper we will apply the developed theoretical approaches to X-ray imaging of the internal structure of a porous material.

Returning to a qualitative analytical study of the field $u(x, z)$ given by equations (12), (13) we have to mark its properties: (a) $|u(x, z \rightarrow 0)|^{2}=1$ for a short distance that is much smaller than the Rayleigh length $a^{2} / \lambda$ and (b) $u(x, z \rightarrow \infty)=1$ for a long distance that is much larger than that length. Following Subsections $4.1-4.4$ present the corresponding approximate expressions for the fields $u(x, z)$ obtained in the cases of large and small values of Fresnel and phase numbers $F$ and $\Phi$.

\subsection{Large Fresnel number $F$}

In this case the critical point $t_{1}=\xi=x / a$ coincides with lateral coordinate that allows us to determine the function $\varphi(\xi)$ of the shape of the pore space interface. The wave field can be derived by substituting $\mathcal{J}(F, \Phi, \xi)$ from (A13) into (13):

$$
\begin{gathered}
u(x, z)=e^{-i \Phi \varphi(\xi)}\left\{1+\frac{\delta z}{2 a}\left[\varphi^{\prime \prime}(\xi)-i \Phi \varphi^{\prime 2}(\xi)\right]\right\} \\
|u(x, z)|^{2}=1+\delta \frac{z}{a} \varphi^{\prime \prime}(\xi)+\frac{\delta^{2}}{4} \frac{z^{2}}{a^{2}}\left\{\left[\varphi^{\prime \prime}(\xi)\right]^{2}+\Phi^{2}\left[\varphi^{\prime}(\xi)\right]^{4}\right\}, \xi=x / a .
\end{gathered}
$$

One can see that the $x$ dependence of the intensity is given by the first and second derivatives of the pore shape function $\varphi(\xi)$. They both would contribute to an edge enhanced image of the pores ${ }^{8}$ described by formula (16). If we discard the third term, we see the intensity distribution at a certain distance from the sample reproduces the second derivative of the field phase on the back plane of the sample. The first two terms in (16) correspond to the results of Ref. ${ }^{17}$ devoted to phase contrast imaging of thin objects but in our work we applied the stationary phase method to not the wave field but the measured intensity directly. Unlike the results of Ref. ${ }^{17}$ formulas (15), (16) contain $\Phi$ and $\varphi^{\prime}$ and offer better characterization of the object. In addition, these formulas can be used to find the derivatives of the pores shape function and phase number $\Phi$ (and, hence, the pore radius $a$ (see (14)) from the measurement of the field behind the sample.

\subsection{Small Fresnel number $F$}

This is the case of long distance $z \gg a^{2} / \lambda$. Large $z$ and low refraction $\delta$ are physically alike, since both variables result in the same weak perturbation of the initial plane wave field $u(x, z)=1$. So, one may write down:

$$
J(F \rightarrow 0, \Phi, \xi) \approx J(F, \Phi \rightarrow 0, \xi) .
$$


This case is considered in more details in Appendix II. Using the expression (A16) for $J(F, \Phi, \xi)$ in (13) gives:

$$
\begin{gathered}
u(x, z)=1-\sqrt{\frac{F}{i}} J_{0}(\Phi)-e^{i \frac{\pi}{4} F^{\frac{3}{2}}}\left\{J_{2}(\Phi)-2 \xi J_{1}(\Phi)+\xi^{2} J_{0}(\Phi)\right\}+\cdots, \xi=\frac{x}{a}, F \ll 1 \\
|u(x, z)|^{2}=1-\sqrt{\frac{2 a^{2}}{\lambda z}} \int_{-\infty}^{\infty} \mathrm{d} t \cdot(\sin [\delta k a \varphi(t)]+1-\cos [\delta k a \varphi(t)])+\cdots,
\end{gathered}
$$

where $J_{0}(\Phi), J_{1}(\Phi)$ and $J_{2}(\Phi)$ are defined in (A17).

\subsection{Large phase number $\Phi$}

Here the phase difference acquired due to a pore space is large. Of course, that is the case of large radius $a$ and relatively soft X-rays because of the value of $\delta$ scales as $\lambda^{2}$. Then, as mentioned in Section 3, the second critical point $t=0$ of the integral (13) plays its role (see Appendix II). By substituting (A21), (A22) into (13) we obtain:

$$
\begin{gathered}
u(x, z)=\exp \left\{i F \pi \xi^{2}-i \Phi\right\} \cdot \sqrt{\frac{a}{\delta\left(-\varphi^{\prime \prime}\right) z}}\left[1+\frac{c(x)}{\Phi}\right], \xi=\frac{x}{a}, \Phi \gg 1 \\
|u(x, z)|^{2}=\frac{a}{\delta\left(-\varphi^{\prime \prime}\right) z}\left[1-\frac{a}{\delta\left(-\varphi^{\prime \prime}\right) z}\right]
\end{gathered}
$$

where $C(x)$ is defined by (A22) and $\left(-\varphi^{\prime \prime}\right)$ is taken at $\xi=0$, that characterizes the pore interface inside the pore space. From (19) and (20) one can see that large phase difference $S(x)$ acquired due to either large pore radius $a$ or large material permittivity decrement $\delta$ leads to a strong distortion of the initial field $u(x, z)$. However, this effect disappears at a large distance from the sample. It should be mentioned that this conclusion is in agreement with electrodynamics and mathematical properties of fast oscillating integrals.

\subsection{Small phase number $\Phi$}

By using formula (A23) of Appendix II and referring to the initial expressions (12), (13) we can obtain:

$$
\begin{aligned}
& u(x, z)=1-\Phi \sqrt{i F} \int_{-\infty}^{\infty} \mathrm{d} t \varphi(t) \exp \left\{i F \cdot \pi\left(t-\frac{x}{a}\right)^{2}\right\}= \\
& =1-i \Phi\left[\sqrt{\frac{F}{i}} \int_{-\infty}^{\infty} \mathrm{d} t \varphi(t) \exp \left\{i F \cdot \pi\left(t-\frac{x}{a}\right)^{2}\right\}\right], \Phi \ll 1 .
\end{aligned}
$$

The term in brackets is the Fresnel integral applied directly to the pore shape function $\varphi(t)$ similar to the field amplitude in the case of absorption contrast. This means that in the case of weak refraction the phase contrast can be regarded as an absorption contrast. Finally, to complete this section we give an expression for the field module:

$$
|u(x, z)|^{2}=1-4 \pi \delta \frac{a}{\lambda} \sqrt{\frac{a^{2}}{z \lambda}} \int_{-\infty}^{\infty} \mathrm{d} t \varphi(t) \cos \left\{\pi \frac{a^{2}}{z \lambda}\left(t-\frac{x}{a}\right)^{2}+\frac{\pi}{4}\right\}
$$

It is easy to verify that this expression at large distance $z$ coincides with (19) for small $\delta$ and yields:

$$
|u(x, z)|^{2}=1-2 \pi \sqrt{2} \delta \frac{a}{\lambda} \sqrt{\frac{a^{2}}{z \lambda}} \int_{-\infty}^{\infty} \mathrm{d} t \varphi(t) .
$$

\section{DISCUSSION}

Many modern methods of studying the internal structure of an object imply two approaches. At the first the probing beam is propagated through a sample containing features like grains, voids, cracks etc. The result is a modified field distribution on the back side of the sample. It can be recorded and processed "as it is", if a detector with high spatial resolution is available. In the simplest form - standard X-ray radiography - this approach has been used in Section 2 to estimate the optimum thickness and the photon number per pixel required for successful pore detection. If the spatial resolution defined by the detector used is not sufficient, X-ray microscope optics ${ }^{*}$ or coherent lensless X-ray imaging is

\footnotetext{
${ }^{*}$ Obviously, in order to restore the pore shape in this case an advanced computer microscopy ${ }^{19}$ is required to process the optical field patterns.
} 
used $^{7}$. Coherent X-ray/EUV imaging is known to consist in recording the diffraction patterns at a definite distance and using the reconstruction algorithms data to restore the field module and phase on the back side of the sample ${ }^{7,9}$.

For X-ray studies of low absorption materials the results of Section 2 cannot be directly used, and phase contrast imaging has no alternative. Therefore, in Section 3 we reduced the pore imaging problem to investigation of a kind of the Fresnel integral (13) for the field in any plane located in the half space behind the sample. The Fresnel integral $J(F, \Phi, \xi)$ has been shown to depend on three dimensionless parameters: $F, \Phi$ and the pores shape function $\varphi(\xi)$.

We developed a set of computer codes ${ }^{* *}$ to calculate $J(F, \Phi, \xi)$ and the amplitude and phase field distributions for a wide range of the input parameters and various types of function $\varphi(\xi)$. In addition, in Section 3 in order to provide a physical understanding of the discussed problems in X-ray investigation of low absorption materials we have presented an analysis of the wave field produced by a coherently illuminated sample containing pore spaces. The obtained formulas (15) - (24) can be of use in choosing detector position and region domains for the computer simulation.

Consider, for example, the optimal position of the detector $z_{o p t}$ for a phase contrast based pore observation with using of formulas (16) and (24), while the field intensity is recorded at small and large distances. It is obvious that due to low absorption contrast the initial intensity near the sample (small $z$ ) is slightly disturbed. On the other hand, at far distances (large $z$ ) the intensity of the initial reference wave, i.e. plane wave, would be dominant, as a field perturbation induced by the object is attenuated. Therefore, there is a location of the detector to observe the maximum phase contrast pattern. To find this location on the optical axis one should equate the formulas (16) and (24) at $x \approx 0$. Then we have:

$$
F_{\text {opt }} \equiv \frac{a^{2}}{\lambda z_{\text {opt }}}=\frac{1}{2}\left[\frac{-\varphi \prime \prime(0)}{\pi \int_{-\infty}^{\infty} \mathrm{d} t \varphi(t)}\right]^{2 / 3}
$$

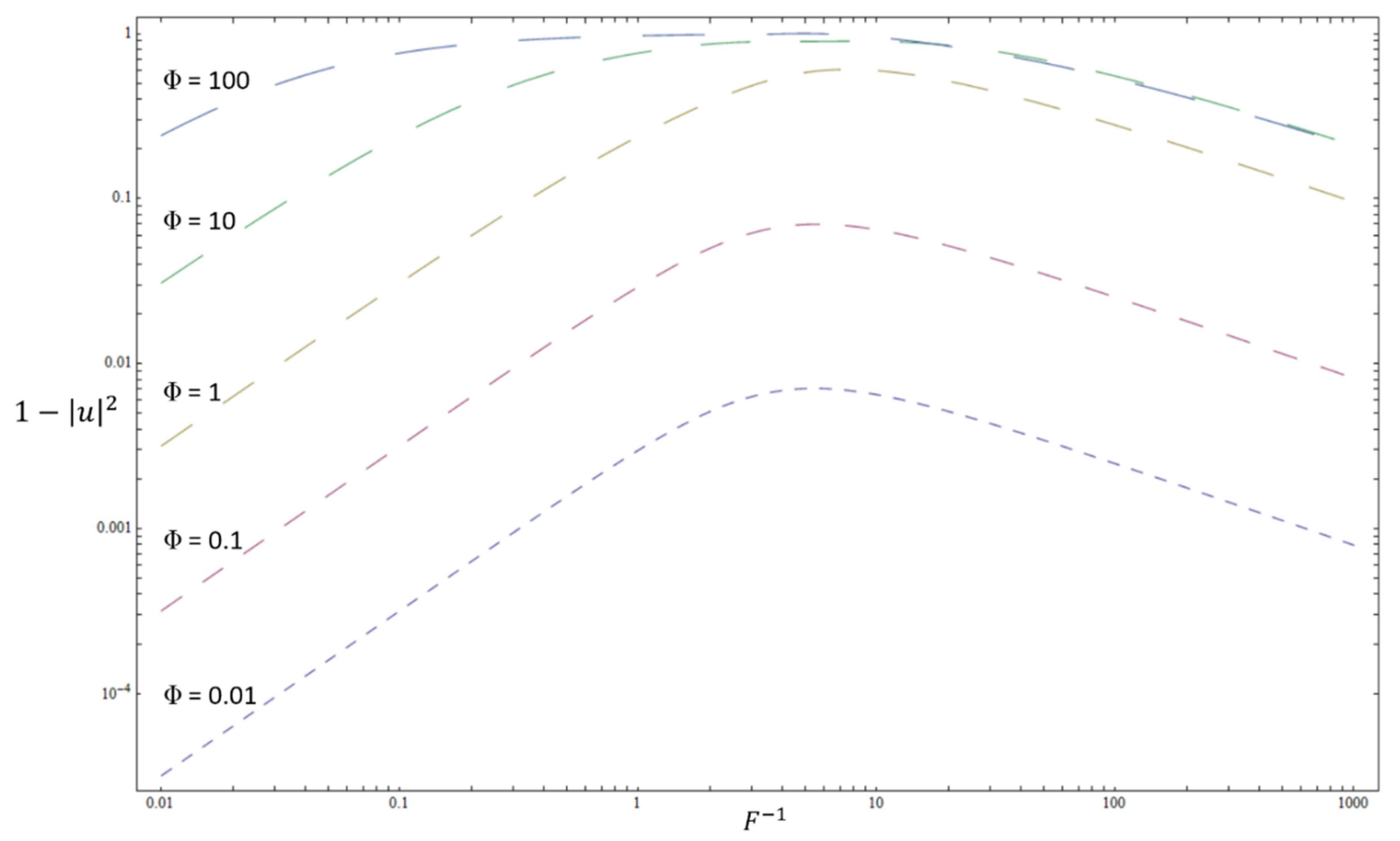

Figure 2. The contrast value $1-|\mathrm{u}(\mathrm{x}, \mathrm{z})|^{2}$ observed on the optical axis $x=0$ by a single pore embedded in a low absorption material as a function of the Fresnel and phase numbers $F\left(F^{-1}=\frac{\lambda z}{a^{2}}\right)$ and $\Phi=2 \pi \delta \frac{a}{\lambda}$. (see Fig.1).The Fresnel integral $J(F, \Phi, \xi)$ was calculated following (13) with the Gaussian pore shape function $\varphi(\xi)$.

Fig. 2 shows that the optimum detector position $z_{\text {opt }}$ corresponds to the Fresnel number $F_{\text {opt }} \sim 1$ with a weak dependence on the decrement $\delta$. The term in brackets demonstrates also a weak dependence on the pore shape function $\varphi(t)$. Then, according to (16) the maximum contrast value with respect to the reference wave intensity can be written as:

** These codes are available also at on-line web form ${ }^{18}$. 


$$
1-\left|u\left(x, z_{o p t}\right)\right|^{2}=\delta \frac{z_{o p t}}{a}\left[-\varphi^{\prime \prime}(0)\right] .
$$

that is proportional to the decrement $\delta$.

Fig. 2 demonstrates the $z$ dependence of the contrast ratio $\frac{1-|u(x, z)|^{2}}{|u(x, z=0)|^{2}}=1-|u(x, z)|^{2}$ on the optical axis $x=0$ calculated for various values of the phase number $\Phi$. The results were obtained with the help of direct computation of the integral $J(F, \Phi, \xi)(13)$ with the Gaussian shape function $\varphi(\xi)=e^{-\xi^{2}}$.

\section{NUMERICAL SIMULATION OF PORE PTYCHOGRAPHY EXPERIMENTS}

As an example, we present an application of ptychographic technique for numerical simulation of the complex wave field after its propagation though ceramic material containing the pores. The ceramics is modelled by $\mathrm{Y}_{3} \mathrm{Al}_{5} \mathrm{O}_{12}$ glass sample containing hundreds of the pores with their random radius in the range from 0.5 to $0.75 \mu$ and random location inside volume of interest $(-4 ; 4) \times(-4 ; 4) \mu \mathrm{m}^{2}$. We use the probing X-ray wavelength $\lambda=0.124 \mathrm{~nm}$, with the refractive index of the modelled glass being $n=1-8.83 \cdot 10^{-6}+i \cdot 1.67 \cdot 10^{-7}$. The pore spaces are assumed to be filled by the air with $n_{o} \approx 1$. A square $385 \times 385$ detector with the pixel size of $50 \mu \mathrm{m}$ is located at the distance of 2 meter from the object. Then, according to Ref. ${ }^{16}$ the object domain size is $5 \times 5 \mu \mathrm{m}^{2}$ with the spatial resolution $13 \mathrm{~nm}$. The X-ray source is set to be a point source collimated by a circular aperture of diameter $5 \mu \mathrm{m}$ near the object. During the ptychography scan the object moves along the spiral trajectory. Thus, the total field of view turns out to be a circle of diameter about $19 \mu \mathrm{m}$ (Fig.3). The results of the simulation are presented in Fig.4 that shows the real (left) and imaginary (right) parts of the restored field directly after the object. All pores are clearly distinguishable, and the best image quality is observed on the regions of pores overlapping.

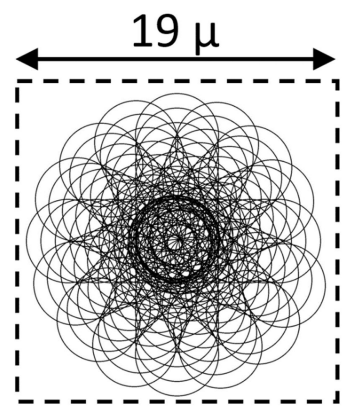

Figure 3. Total field of view composed of 128 circles $5 \mu \mathrm{m}$ in diameter on a spiral trajectory.

A similar numerical experiment with a single pore determines the minimum detectable pore diameter as $a_{0}=4.8 \mu \mathrm{m}$. To find the related minimum detectable concentration of the pores with a typical radius of $0.5 \mu \mathrm{m}$ we carried out additional simulations with the same geometric parameters and 10 random sampling of the pores. The result obtained is 55 pores inside $8 \times 8 \mu \mathrm{m}^{2}$ area or $0.86 \mu \mathrm{m}^{-2}$. Given the ceramic sample is of $1-\mathrm{cm}$ thickness, the minimum detectable concentration of the pores is found to be $\left(0.86^{*} 10^{8}\right) / 1=0.86^{*} 10^{8} \mathrm{~cm}^{-3}$.

\section{SUMMARY}

1. We demonstrated that there is the optimal sample thickness maximizing the signal-to-noise ratio in pore observation experiments based on X-ray absorption contrast.

2. We introduced a model for characterization of pores in transparent materials in coherent X-ray radiography data. The model allows us to determine the pore size $a$ and its shape function $\varphi(\xi)$. 


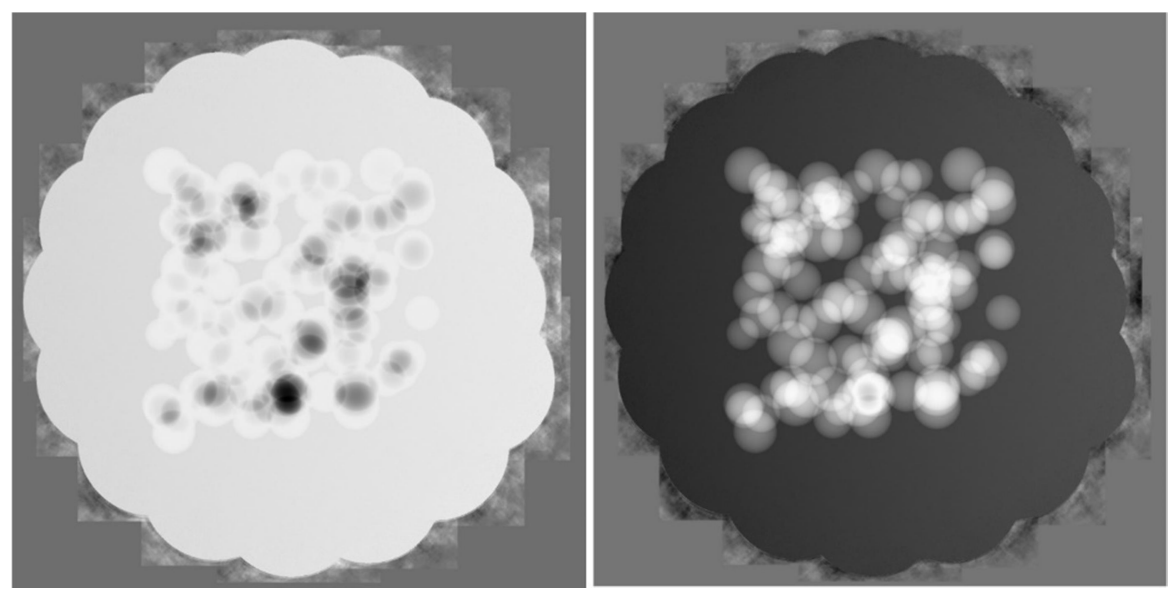

Figure 4. Simulated real (left) and imaginary (right) parts of restored wave field created by $\mathrm{Y}_{3} \mathrm{Al}_{5} \mathrm{O}_{12}$ sample containing 100 pores.

3. The structure of the field after propagation through a sample containing the pores is expressed by the Fresnel integral similar to that of in-line holography. The suggested model depends on two dimensionless parameters: the Fresnel number $F=a^{2} \lambda z$ and the phase number $\Phi=a k \delta$. A measurement of the field intensity with a proper position of the detector makes it possible to determine the pore size $a$ and its shape function $\varphi(\xi)$.

4. We have determined four spatial and frequency domains for significant simplification of the Fresnel integral. They correspond to four values of the mentioned parameters: $F \gg 1, F \ll 1, \Phi \gg 1$ and $\Phi \ll 1$.

5. The analysis of these four cases points out to the optimal position of the detector to achieve the best contrast in X-ray study of the pores (formulas (25) and (26)).

6. In the case of a Gaussian function $\varphi(\xi)$ we have carried out a series of numerical simulation of the X-ray imaging experiments. The software developed for calculating of the coherent fields in X-ray studies of objects with an arbitrary pore function is available in the public domain.

The authors are grateful to Dr.E.A. Cheshev and Dt.I.M.Tupitsyn for useful and stimulating discussions. This work was supported in part by the Russian Foundation of Basic Research projects 19-02-00394, 18-08-01066 and 18-29-17039.

\section{REFERENCES}

[1] Nagai, K., Musgrave, C. S. A. and Nazarov, W., "A review of low density porous materials used in laser plasma experiments," Physics of Plasmas, 25(3), 030501 (2018).

[2] Messing, G. L. and Stevenson, A. J., "Toward Pore-Free Ceramics," Science, 322(5900), 383-384 (2008).

[3] Rouquerol, F., Sing, K. S. W. and Rouquerol, J., [Adsorption by Powders and Porous Solids Principles, Methodology, and Applications], Academic Press, San Diego (1999).

[4] Lowell, S., Shields, J. E., Thomas, M. A. et al., [Characterization of Porous Solids and Powders: Surface Area, Pore Size and Density], Springer Science+Business Media, New York (2004).

[5] Anovitz, L. M. and Cole, D. R., "Characterization and Analysis of Porosity and Pore Structures," Reviews in Mineralogy and Geochemistry, 80(1), 61-164 (2015).

[6] Melnichenko, Y. B., [Structural Characterization of Porous Materials Using SAS], Springer International Publishing, Cham, 139 - 171 (2016).

[7] Jacobson, C., [X-ray Microscopy], Cambridge University Press, Cambridge (2020).

[8] Mayo, S. C., Stevenson, A. W. and Wilkins, S. W., "In-line phase-contrast X-ray imaging and tomography for materials science," Materials, 5(5), 937-965 (2012).

[9] Pfeiffer, F., "X-ray ptychography," Nature Photonics, 12(1), 9-17 (2018). 
[10] Tsai, E. H., Billaud, J., Sanchez, D. F. et al., "Correlated X-ray 3D ptychography and diffraction microscopy visualize links between morphology and crystal structure of lithium-rich cathode materials," Iscience, 11, 356365 (2019).

[11] Paganin, D., [Coherent X-Ray Optics], Oxford University Press on Demand (2006).

[12] Paganin, D. M. and Pelliccia, D., "Tutorials on X-ray phase contrast imaging: Some fundamentals and some conjectures on future developments," arXiv Preprint arXiv:1902.00364 (2019). https://arxiv.org/abs/1902.00364.

[13] Snigirev, A., Snigireva, I., Kohn, V. et al., "On the possibilities of x-ray phase contrast microimaging by coherent high-energy synchrotron radiation," Review of Scientific Instruments, 66(12), 5486-5492 (1995).

[14] Argunova, T. S. and Kohn, V. G., "Study of micropores in single crystals by in-line phase contrast imaging with synchrotron radiation," Physics-Uspekhi, 62(6), 602 (2019).

[15] Rodenburg, J. and Maiden A., [Springer Handbook of Microscopy], Springer International Publishing, Cham 819-904 (2019).

[16] Popov, N. L., Artyukov, I. A., Vinogradov, A. V. and Protopopov, V. V., "Wave packet in the phase problem in optics and ptychography," Physics-Uspekhi, 63, 766-774 (2020).

[17] Schelokov, I., Weitkamp, T. and Snigirev, A., "Reconstruction of an object phase transmission function from in-line X-ray holograms," Optics Communications, 213(4-6), 247-258 (2002).

[18] http://77.51.206.116:65000/fiber_web/x-ray_porosimetry.aspx.

[19] Murphy, R., "The quest for quantitative microscopy," Nat Meth, 9, 627-627 (2012)

[20] Erdelyi, A., [Asymptotic Expansions], Dover Publications (1956).

[21] Papoulis, A., [Systems and Transforms with Applications in Optics], Krieger Pub. Co. (1981).

[22] Olver, F.W.J, [Introduction to Asymtotics and Special Functions], Academic Press, New York (1974).

[23] Guillemin, V. and Sternberg, S., [Geometric asymptotics], American Mathematical Soc., (1990).

[24] Fedoryuk, M., [The Method of Steepest Descent] Nauka, Moscow, (1977) (in Russian).

[25] Bogoliubov, N. and Shirkov, D., [Quantum fields], Benjamin \& Cummings Pub. Co Inc, (1982).

\section{APPENDIX I. ASYMPTOTIC EXPANSION OF ONE TYPE OF FAST OSCILLATING INTEGRAL}

Let us consider the integral

$$
J(F)=\int_{-\infty}^{\infty} \exp \{i F \cdot f(t)-i \psi(t)\} \mathrm{d} t
$$

in the case of large $F$, with $t_{0}$ being critical point, i.e. $\frac{d f(t)}{d t}=0$ at $t=t_{0}$ and

$$
\begin{gathered}
f(t)=f+\frac{f^{\prime \prime}}{2}\left(t-t_{0}\right)^{2}+\frac{f^{\prime \prime \prime}}{6}\left(t-t_{0}\right)^{3}+\frac{f^{\prime \prime \prime \prime}}{24}\left(t-t_{0}\right)^{4}+\cdots, f^{\prime}=0, f^{\prime \prime}>0 \\
\psi(t)=\psi+\psi^{\prime}\left(t-t_{0}\right)+\frac{\psi^{\prime}}{2}\left(t-t_{0}\right)^{2}+\cdots
\end{gathered}
$$

where $f, f^{\prime} \ldots$ and $\psi, \psi^{\prime} \ldots$ are the values of functions $f(t)$ and $\psi(t)$ and their derivatives at $t=t_{0}$. The solution of the problem described by (A1) - (A3) is used in Section 3 and Appendix II to evaluate the pore imaging integral $J(F, \Phi, \xi)(13)$ in the case of large Fresnel and phase numbers.

The integral (A1) belongs to a class of fast oscillating integrals well-known in mathematical literature. If functions $f(t)$ and $\psi(t)$ have no singularities, (A1) can be simplified by using the first term of its asymptotic expansion into power series of inverse $\sqrt{F}$. This is the stationary phase method which is described in textbooks ${ }^{20-22}$. However, if functions $f(t)$ and $\psi(t)$ have any singularities one needs considering higher powers of $F^{-1 / 2}$, as, for example, in the case of the presented paper. The related solution can be found in special mathematical literature ${ }^{23}, 24$. Since we often use the asymptotic expansion of the integral (A1), we would like to present a brief discussion of the expressions obtained for the first few terms of this expansion.

By substituting $y=\sqrt{F}\left(t-t_{0}\right)$ into (A1) and expanding the exponent into series of $F^{-1 / 2}$ we can write:

$$
F \cdot f(t)-\psi(t)=F f-\psi+\frac{f^{\prime \prime}}{2} y^{2}+\frac{A(y)}{\sqrt{F}}+\frac{B(y)}{F}+\cdots, \quad t \rightarrow t_{0}
$$




$$
A(y)=\frac{f^{\prime \prime \prime}}{6} y^{3}-\psi^{\prime} y, \quad B(y)=\frac{f^{\prime \prime \prime}}{24} y^{4}-\frac{\psi^{\prime \prime}}{2} y^{2}, y=\sqrt{F}\left(t-t_{0}\right) .
$$

Using (A4), (A5) in (A1) enables us to expand the integrand also into a series of $F^{-1 / 2}$ and to reduce the initial integral to the following form:

$$
J(F)=\frac{\exp (i F \cdot f-i)}{\sqrt{F}} \int_{-\infty}^{\infty}\left\{1+\frac{i}{\sqrt{F}} A(y)+\frac{i}{F}\left[B(y)+\frac{i}{2} A^{2}(y)\right]+\cdots\right\} \exp \left\{i \cdot \frac{f^{\prime \prime}}{2} y^{2}\right\} \mathrm{d} y
$$

where the first term corresponds to the stationary phase approximation ${ }^{20}$. In contrast to Ref. ${ }^{20}$ formula (A6) includes also the terms of the order $F^{-1 / 2}$ and $F^{-1}$ that are responsible for the phase contrast needed for the X-ray pore investigation. To find the corresponding coefficients we can reduce the integrals in (A6) to Gaussian quadratures and their derivatives ${ }^{25}$ :

$$
I(\alpha+i \eta, \beta)=\int_{-\infty}^{\infty} \exp \left[i\left(\alpha y^{2}+2 \beta y\right)-\eta y^{2}\right] \mathrm{d} y=\sqrt{\frac{i \pi}{\alpha}} \exp \left(-i \frac{\beta^{2}}{\alpha}\right), \alpha>0, \eta \rightarrow+0
$$

with:

$$
\begin{gathered}
\int_{-\infty}^{\infty} \exp \left[i \alpha y^{2}\right] y^{2} \mathrm{~d} y=\frac{i}{2 \alpha} \sqrt{\frac{i \pi}{\alpha}}, \int_{-\infty}^{\infty} \exp \left[i \alpha y^{2}\right] y^{4} \mathrm{~d} y=-\frac{3}{4 \alpha^{2}} \sqrt{\frac{i \pi}{\alpha}} \\
\int_{-\infty}^{\infty} \exp \left[i \alpha y^{2}\right] y^{6} \mathrm{~d} y=-\frac{15 i}{8 \alpha^{3}} \sqrt{\frac{i \pi}{\alpha}}
\end{gathered}
$$

Now one can see that formula (A6) with $A(y)$ and $B(y)$ defined in (A4) and (A5) after the integration over $y$ provides the desired approximation of (A1) in the case of large Fresnel numbers ${ }^{*}$

$$
J(F)=\int_{-\infty}^{\infty} \exp \{i F \cdot f(t)-i \psi(t)\} \mathrm{d} t=\frac{\exp (i F \cdot f-i \psi)}{\sqrt{F}} \sqrt{\frac{2 i}{f^{\prime \prime}}}\left(1+\frac{C}{F}\right), F \gg 1
$$

where

$$
C\left(t_{0}\right)=\frac{5 i\left(f^{\prime \prime \prime}\right)^{2}}{24\left(f^{\prime \prime}\right)^{3}}-\frac{3}{\left(f^{\prime \prime}\right)^{2}}\left(\frac{i f^{\prime \prime \prime \prime}}{24}+\frac{f^{\prime \prime \prime} \psi^{\prime}}{6}\right)+\frac{\psi^{\prime \prime}-i\left(\psi^{\prime}\right)^{2}}{2 f^{\prime \prime}}
$$

Note that in (A4) and (A5) $f, f^{\prime} \ldots$ and $\psi, \psi^{\prime} \ldots$ are the functions $f(t)$ and $\psi(t)$ and their derivatives at $t=t_{0}$.

\section{APPENDIX II. THE PROPERTIES OF THE FRESNEL INTEGRAL $J(F, \Phi, \xi)$}

The results of Appendix I allow us to investigate the Fresnel integral $J(F, \Phi, \xi)$ in the cases of large and small values of the Fresnel and phase numbers in application of our problem of X-ray pore studies.

\section{(a) Large Fresnel number}

We use formulas (A9), (A10) to simplify the integral $J(F, \Phi, \xi)$ of the pore imaging (see Sec. 2, formula (13)) as:

$$
\begin{gathered}
f(t)=\pi(t-\xi)^{2}, t_{0}=\xi, f^{\prime \prime}=2 \pi, f^{\prime \prime \prime}=0, f^{\prime \prime \prime \prime}=0 \\
\psi(t)=\Phi \varphi(t), \psi=\Phi \varphi, \psi^{\prime}=\Phi \varphi^{\prime}, \psi^{\prime \prime}=\Phi \varphi^{\prime \prime}
\end{gathered}
$$

and to get the following expression:

$$
J(F, \Phi, \xi)=\frac{\sqrt{i} \exp (-i \Phi \varphi)}{\sqrt{F}}\left[1+\frac{\Phi}{4 \pi F}\left(\varphi^{\prime \prime}-i \Phi \varphi^{\prime 2}\right)\right], \quad F \gg 1
$$

where $F$ and $\Phi$ are the Fresnel and phase numbers, $\varphi, \varphi^{\prime}$ and $\varphi^{\prime \prime}$ are the pore shape function and its derivatives taken at $t=\xi=\frac{x}{a}, x$ is the lateral coordinate and $a$ is the pore radius.

\footnotetext{
${ }^{*}$ Note that there are only terms containing even powers of $y$ in the integrand.
} 


\section{(b) Small Fresnel number}

This is the case of large distance $z$ that corresponds to the first order perturbation theory, i.e. a pore induces the field fluctuation against the background of primary plane wave. The value of the phase number $\Phi$ is not relevant. Therefore, in this case one can expect $u(x, z)=1$ in (13) and, hence:

$$
J(F \rightarrow 0, \Phi, \xi) \approx \sqrt{\frac{i}{F}}
$$

To investigate the field $u(x, z)$ at small numbers $F$ with a higher accuracy it is convenient to rewrite the Fresnel integral $J(F, \Phi, \xi)(13)$ in the form:

$$
J(F, \Phi, \xi)=\sqrt{\frac{i}{F}}-\int_{-\infty}^{\infty} \mathrm{d} t\left[\exp \left\{i F \cdot \pi(t-\xi)^{2}\right\}\right]\left[1-e^{-i \Phi \varphi(t)}\right]
$$

If the function $\varphi(t)$ decreases fast enough for $|t|>a$, the first multiplier of the integrand in (A15) can be expanded in the power series of $F$. Then:

$$
J(F, \Phi, \xi)=\sqrt{\frac{i}{F}}-J_{0}(\Phi)-i F \pi\left\{J_{2}(\Phi)-2 \xi J_{1}(\Phi)+\xi^{2} J_{0}(\Phi)\right\}
$$

where:

$$
\begin{gathered}
J_{0}(\Phi)=\int_{-\infty}^{\infty} \mathrm{d} t\left[1-e^{-i \Phi}(t)\right], \quad J_{1}(\Phi)=\int_{-\infty}^{\infty} t \mathrm{~d} t\left[1-e^{-i \Phi \varphi(t)}\right], \\
J_{2}(\Phi)=\int_{-\infty}^{\infty} t^{2} \mathrm{~d} t\left[1-e^{-i \Phi \varphi(t)}\right] .
\end{gathered}
$$

Formulas (A16), (A17) together with (13) can be used to evaluate the field $u(x, z)$ for small Fresnel numbers $F$ within the accuracy of the second order perturbation theory.

\section{(c) Large phase number}

To estimate the Fresnel integral (13) we refer again to Appendix I, with taking into account the second critical point $t_{2}=0$ of $J(F, \Phi, \xi)(13)$ and large number $\Phi$. This results in the following substitution for $J(F)$ in formula (A1):

$$
F=\Phi, f(t)=-\varphi(t), t_{0}=0, \psi(t)=-F \pi(t-\xi)^{2}
$$

Then we can write:

$$
\begin{gathered}
F=\Phi, f=-1, f^{\prime \prime}=-\varphi^{\prime \prime}(0)>0, f^{\prime \prime \prime}=0, f^{\prime \prime \prime \prime}=-\varphi^{\prime \prime \prime \prime}(0), \\
\psi=-F \pi \xi^{2}, \psi^{\prime}=2 F \pi \xi, \psi^{\prime \prime}=-2 F \pi,
\end{gathered}
$$

into (A9), (A10) and after simple manipulations we obtain the desired formula for the Fresnel integral:

$$
\begin{gathered}
J(F, \Phi, \xi)=\sqrt{\frac{i}{\Phi}} \exp \left\{i F \pi \xi^{2}-i \Phi\right\} \cdot \sqrt{\frac{2 \pi}{-\varphi^{\prime \prime}}}\left[1+\frac{C(x)}{\Phi}\right], \xi=\frac{x}{a}, \Phi \gg 1 \\
C(x)=\frac{1}{-\varphi \prime \prime}\left[\frac{i \varphi^{\prime \prime \prime \prime \prime}}{8\left(-\varphi^{\prime \prime \prime}\right)}-F \pi\left(1+i 2 F \pi \xi^{2}\right)\right]
\end{gathered}
$$

Note that according (A18) - (A20) the function $\varphi(t)$ and its derivatives are taken at the critical point $t_{0}=0$ of the integral (A1).

\section{(d) Small phase number}

In this case the form (A15) of the Fresnel integral is more appropriate than (13). Expanding it into a series by $\Phi$ and using the first two terms we can write:

$$
J(F, \Phi, \xi)=\sqrt{\frac{i}{F}}-i \Phi \int_{-\infty}^{\infty} \varphi(t) d t \exp \left\{i F \cdot \pi(t-\xi)^{2}\right\}, \xi=\frac{x}{a} .
$$

Formula (A23) demonstrates the evident fact: the Fresnel transform of a phase signal $\exp \{i \varphi(t)\}$ can be reduced to the Fresnel transform of its phase $\varphi(t)$. 